\title{
Congenital Pelvic Arteriovenous Malformation Presenting with Urinary Retention: A Case Report
}

\author{
Fayez T. Hammad ${ }^{a}$ Fahmy Shawish $^{\mathrm{b}}$ Essa Kazim $^{\mathrm{b}}$ \\ a Department of Surgery, Faculty of Medicine and Health Sciences, United Arab Emirates University, Al Ain, and \\ ${ }^{b}$ Department of Urology, Dubai Hospital, Dubai, United Arab Emirates
}

\section{Key Words}

Pelvic arteriovenous malformations • Urinary retention •

Computed tomography scan

\begin{abstract}
Objective: To report a case of congenital pelvic arteriovenous malformation (pAVM) with urinary retention. Clinical Presentation and Intervention: A 39-year-old male patient presented with inability to void urine for $8 \mathrm{~h}$. He did not have a history of dysuria or fever, but had increasing urinary frequency and difficulty in voiding over 2 years. Examination revealed a suprapubic mass without external signs of vascular anomaly. A huge, soft, irregular, non-pulsating mass was felt rectally. This mass did not disappear on urinary bladder decompression. Subsequent ultrasound and CT scan revealed pAVMs filling most of the pelvic cavity. Conclusion: To our knowledge, this is the first report of a male patient with congenital pAVM presenting with urinary retention, emphasizing the need for a high index of suspicion for $p A V M$ to avoid potential severe bleeding during suprapubic catheterization for urinary drainage.
\end{abstract}

Copyright $\odot 2011$ S. Karger AG, Basel

\section{KARGER}

Fax +4161306 1234

E-Mail karger@karger.ch

www.karger.com (c) 2011 S. Karger AG, Basel

1011-7571/11/0203-0294\$38.00/0

Accessible online at:

www.karger.com/mpp

\section{Introduction}

Pelvic arteriovenous malformation (pAVM) is rare, especially in male patients [1]. In this condition, the vascular lesion is formed by multiple abnormal communications between the arterial and $\Delta$ venous systems [2]. These pAVMs, or fistulas with a similar appearance, are usually secondary to neoplasms, pelvic trauma, or surgical procedures and more rarely can be of congenital origin [1].

pAVMs may result in a variety of symptoms such as pelvic pain, erectile dysfunction and haematuria, but have not, to our knowledge, been reported to present primarily with urinary retention [1]. We herein present some CT images of a case of congenital pAVM in a male patient who presented with urinary retention, emphasizing the importance of having a high index of suspicion for pAVM, as suprapubic catheterization could result in severe haemorrhage and a catastrophic outcome.

\section{Case Report}

A 39-year-old lean patient presented with inability to void for $8 \mathrm{~h}$. He gave no history of dysuria or fever, but had increasing urinary frequency and difficulty in voiding over 2 years. There was no history of trauma and his past surgical and medical history was non-contributory.
Fayez T. Hammad, FRCSEd, PhD, FRACS (Urology)

Department of Surgery, Faculty of Medicine and Health Sciences PO Box 17666, Al Ain (United Arab Emirates)

Tel. +971 50488 0021, Fax +971 37672067

E-Mail fayezh@uaeu.ac.ae 


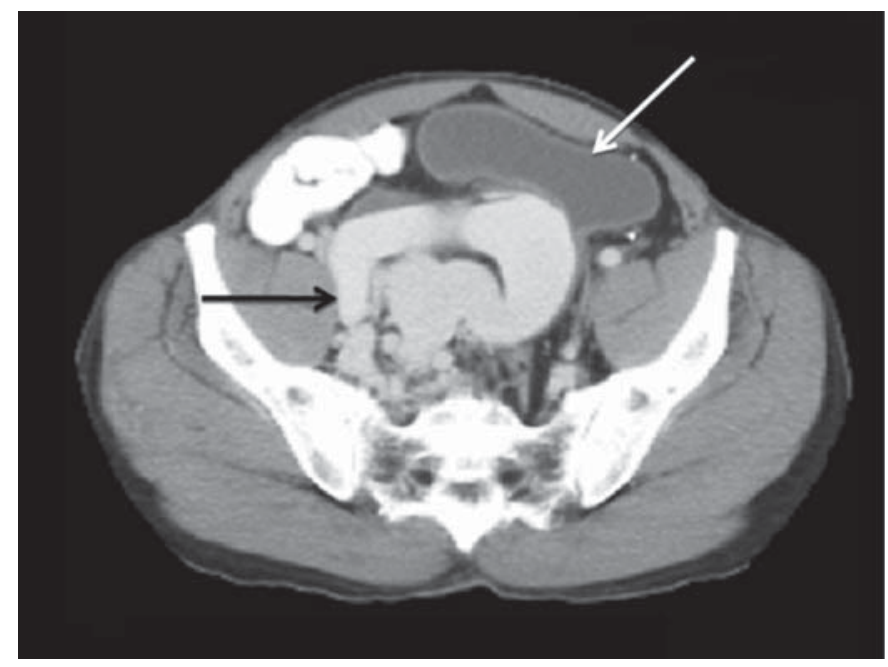

Fig. 1. CT scan of the pelvis showing the pAVMs (dark arrow) and the urinary bladder which was shifted cranially (light arrow).

On examination, there was a visible, smooth and slightly tender suprapubic mass extending up to 6-7 $\mathrm{cm}$ above the symphysis pubis, suggesting a full urinary bladder. On subsequent examination, no bruit could be heard over the area. The external genitalia were normal with no evidence of varicoceles or other vascular anomalies. Lower limbs did not show any sign of oedema or leg varicosities. Digital rectal examination revealed a small, benign and non-tender prostate but there was a huge, soft, irregular nonpulsating mass in the rectovesical pouch.

A 16-french Foley catheter was inserted easily and drained more than $700 \mathrm{ml}$ of clear urine, but the size of the suprapubic mass did not change significantly. Both urinalysis and culture were negative. Blood investigations including haemoglobin and serum creatinine were normal.

Ultrasonography revealed a mildly dilated right pelvicalyceal system associated with the right hydroureter and a normal-looking left system. It also showed a smooth urinary bladder wall with no evidence of intravesical lesions. In addition, there were dilated tortuous cystic lesions measuring up to $9.5 \mathrm{~cm}$ in diameter, representing dilated pelvic vessels and filling most of the pelvis and extending to the rectovesical pouch. Doppler ultrasonography demonstrated a slow flow of blood in the dilated vessels. A CT scan confirmed the presence of pAVMs which were filling most of the pelvic cavity and surrounding the bladder neck, causing a cranial shift of the urinary bladder and dilatation of the right urinary tract (fig. 1, 2). No abdominal or pelvic lymphadenopathy was noticed.

The patient voided successfully upon the removal of the catheter but the lower urinary tract symptoms persisted. He was therefore offered an angiography and embolization of the feeding vessels, but declined the treatment during the follow-up period of 12 months.

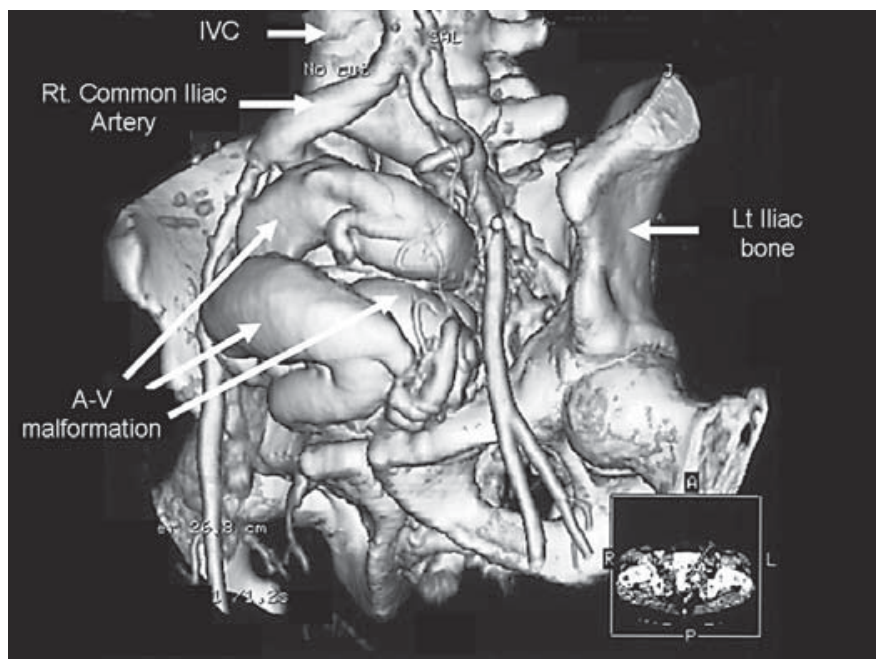

Fig. 2. Three-dimensional CT scan image of the pelvis showing the pAVMs filling most of the pelvis. IVC = Inferior vena cava; $\mathrm{A}-\mathrm{V}=$ arteriovenous; $\mathrm{Rt}=$ right; $\mathrm{Lt}=$ left.

\section{Discussion}

Arteriovenous malformations (AVMs) form when numerous dysplastic arteries immediately drain or shunt into arterialized veins without an intervening normal capillary network [2]. In the pelvis, congenital AVMs are rare, especially in male patients. Indeed, to our knowledge, only 20 cases of congenital pAVM in males have been reported in the English literature from 1966 to January 2010, based on a Medline search using the following keywords: arteriovenous malformation; MeSH terms: arteriovenous malformation and pelvis $[1,3-5]$. Nor did we find any reported case presenting primarily with urinary retention. In male patients, $\mathrm{pAVM}$ presents with various symptoms such as pelvic discomfort, erectile dysfunction, dysuria and haematuria [1].

In this report, we present a case of congenital pAVM in a male patient who presented with urinary retention, probably by exerting extrinsic pressure on, or stretching the bladder neck, similar to other pelvic masses regardless of their aetiology [6]. In the current case, the absence of associated clinical clues, such as scrotal or lower limb vascular anomalies, made it difficult to identify the vascular nature of such a huge, visible, palpable and rectally felt mass. The persistence of the mass following bladder drainage led to the performing of a pelvic ultrasound, which confirmed its vascular nature. The scan showed a mass occupying most of the bony pelvic space, compressing the bladder and the right lower ureter, causing uri- 
nary retention and dilatation of the right system, respectively.

The importance of having a high index of suspicion to recognize this condition cannot be overemphasized, especially in patients who require the introduction of a suprapubic catheter due to the presence of a co-existing impassable outlet obstruction, such as urethral stricture or a severely obstructing prostate. Under these conditions, the introduction of a suprapubic catheter could result in profuse bleeding and a possible catastrophic outcome. To avoid such consequences it is prudent, especially in suspicious cases (prior to the insertion of the suprapubic catheter), to routinely insert a fine needle, under local anaesthesia, along the same tract. It is important to make sure that only urine is aspirated through the fine needle from the suprapubic catheter tract. Performing a diagnostic pelvic sonography before suprapubic catheterization can be helpful.

In symptomatic patients, the pAVM can be treated surgically [7] or with embolization $[1,8]$. Surgical treatment includes ligature of the feeding vessels or excision of the affected area $[9,10]$. Simple vessel ligation may be of no value as new collaterals usually develop and bypass the ligature. Excision of the vessels and the affected area is fraught with complications such as severe haemorrhage or adjacent organ injury $[1,9,10]$. Therefore, angiographic embolization currently represents the treatment of choice for most cases. Nevertheless, the recurrence of the lesions is possible; Jacobowitz et al. [8] reported a mean embolization rate of 2.4 per patient. In the current report, our patient, although symptomatic, declined any intervention and opted for conservative treatment.

\section{Conclusion}

This report presents, to our knowledge, a first case of congenital pAVM in a male patient presenting with urinary retention, emphasizing the need to have a high index of suspicion for pAVM to avoid potential severe bleeding during suprapubic catheterization for urinary drainage.

\section{References}

1 Game X, Berlizot P, Hassan T, Joffre F, Chokairi S, Houlgatte A, Rischmann P: Congenital pelvic arteriovenous malformation in male patients: a rare cause of urological symptoms and role of embolization. Eur Urol 2002;42:407-412.

-2 Legiehn GM, Heran MK: Classification, diagnosis, and interventional radiologic management of vascular malformations. Orthop Clin North Am 2006;37:435-474, vii-viii.

-3 Ishii T, Higashionna T, Hiratsuka Y: Transrectal ultrasound findings of pelvic arteriovenous malformation. J Urol 2001;166:999.
4 Suzuki K, Nishimi D, Morioka H, Takanami M: Hematospermia associated with congenital arteriovenous malformation of internal iliac vessels. Int J Urol 2007;14:370-372.

-5 Kassardjian Z, Lebret T, Mellot F, Herve JM, Barre P, Lugagne PM, Scherrer A, Botto H: Major complex pelvic arteriovenous malformation in a patient with Down syndrome. Urol Int 2002;69:145-149.

6 Doran J, Roberts M: Acute urinary retention in the female. Br J Urol 1975;47:793-796.

$\checkmark 7$ Mimata H, Tanigawa T, Imagawa M, Takahashi S, Yoshio N, Ogata J, Hadama T, Muramoto S: Congenital pelvic arteriovenous fistula in a male. A case report (in Japanese). Nippon Hinyokika Gakkai Zasshi 1992;83: 243-246.
-8 Jacobowitz GR, Rosen RJ, Rockman CB, Nalbandian M, Hofstee DJ, Fioole B, Adelman MA, Lamparello PJ, Gagne P, Riles TS: Transcatheter embolization of complex pelvic vascular malformations: results and long-term follow-up. J Vasc Surg 2001;33:5155.

$>9$ Trout HH 3rd, McAllister HA Jr, Giordano JM, Rich NM: Vascular malformations. Surgery 1985;97:36-41.

10 Pritchard DA, Maloney JD, Bernatz PE, Symmonds RE, Stanson AW: Surgical treatment of congenital pelvic arteriovenous malformation. Mayo Clin Proc 1978;53:607-611. 